\title{
Are item-level strategy shifts abrupt and collective? Age differences in cognitive skill acquisition
}

\author{
DAYNA R. TOURON \\ Appalachian State University, Boone, North Carolina
}

\begin{abstract}
Item-level analysis allows for the examination of qualitative age and individual differences in skill acquisition, which are obscured when aggregating data across items. In the present study, item-level strategy shifts were generally gradual and variable, rather than abrupt and collective. Strategy shift reversions were frequent, and the total transition space was extensive, for both younger and older adults. Shift indices were highly variable between items for both younger and older adults. Age differences in item-level shift patterns suggest that older adults' greater conservatism in strategy selection leads to more gradual strategy shift transitions for individual items as well as to more collective strategy shifts.
\end{abstract}

Age differences in skill and information acquisition are documented across various cognitive domains. Older adults typically learn more slowly than younger adults, and reach lower levels of asymptotic performance (see, e.g., Bosman \& Charness, 1996; Charness, 1981; Hoyer, Cerella, \& Onyper, 2003; Siegler \& Lemaire, 1997; Strayer \& Kramer, 1994; Touron \& Hertzog, 2004a, 2004b; Touron, Hoyer, \& Cerella, 2001, 2004). Several theoretical approaches have been advanced to account for these performance deficits, including differences in strategy choice and efficiency (Strayer \& Kramer, 1994; Touron \& Hertzog, 2004a, 2004b), associative learning deficits (Hoyer et al., 2003; Touron et al., 2001, 2004), and the influence of age-related slowing on component task process (Salthouse, 1994; Touron et al., 2004).

Age differences are particularly pronounced for skill acquisition tasks involving a transition in response method-referred to here as a strategy shift-from performing the task based on an algorithm to retrieving solutions directly from memory (see, e.g., Logan, 1988; Rickard, 1997). Several studies have shown that older adults' strategy shift is slower and less complete than strategy shift by younger adults. This is true for verification and production tasks involving a shift from a computation algorithm to memory retrieval (Touron et al., 2001, 2004), for tasks involving a shift from a simpler visual search algorithm to memory retrieval (Rogers \& Gilbert, 1997; Rogers, Hertzog, \& Fisk, 2000; Touron \& Hertzog, 2004a, 2004b), and for tasks that involve the development of au-

The author extends special thanks to Christopher Hertzog and the reviewers for insightful comments on an earlier version of the manuscript, as well as to Jarrod Hines, Zach Speagle, and Elizabeth Swaim for their assistance with subject recruitment and data collection. This research was supported by National Institute on Aging Grant R01 AG024485. Correspondence concerning this article should be addressed to D. R. Touron, Department of Psychology, Appalachian State University, Boone, NC 28608 (e-mail: tourond@appstate.edu). tomaticity in memory search tasks (Fisk \& Rogers, 1991; Strayer \& Kramer, 1994).

Previous investigations concerning age differences in strategic skill acquisition have focused exclusively on overall task performance. However, an item-level approach could expose further qualitative differences in task approach by older and younger adults that are obscured when analyzing aggregate data. Although such strategies cannot completely account for cognitive performance (Light, 1996; Salthouse, 1991), strategic behavior has a marked impact on performance differences in many complex cognitive tasks (see, e.g., Dunlosky \& Hertzog, 2001; Lemaire \& Arnaud, 2002). Furthermore, aggregation of acquisition data from individual participants into group functions can produce artifactual biases and obscure meaningful regularities (see Estes \& Maddox, 2005; Myung, Kim, \& Pitt, 2000), and analogous consequences may result when data are aggregated across items.

The present study examines age differences in itemlevel strategy shifts using the noun pair (NP) lookup task. Participants were asked to verify whether or not a target noun pair appeared in a lookup table (Ackerman \& Woltz, 1994). Noun pairs were consistently mapped (CM; Shiffrin \& Schneider, 1977) to allow learning of the associative pairings. Discrimination of matched from unmatched pairs could be achieved by either visual search of the lookup table (referred to here as scanning) or, with repetition, by memory retrieval. Because retrieval is typically much faster, strategy shift can be inferred when CM noun pair response times (RTs) become reliably shorter than variably mapped (VM) noun pair RTs (Ackerman \& Woltz, 1994; Rogers \& Gilbert, 1997; Rogers et al., 2000).

In order to facilitate direct examination of item-level strategy shifts, the present study instead employed strategy self-reports following each trial. Strategy reports in such skill acquisition tasks appear to be valid reflections of strategy implementation. RT distributions are distinct between reports of algorithm-based versus retrieval-based 
responding (Hoyer, Cerella, \& Onyper, 2003; Rickard, 2004; Touron \& Hertzog, 2004a; Touron et al., 2004), and reported algorithm trials demonstrate characteristics of effortful processing, such as addend effects, which are absent in the more fluent retrieval report trials (see Hoyer et al., 2003). ${ }^{1}$

Recent evidence examining age differences in strategy reports during NP task performance has indicated that subjective choice factors influence observed age differences in performance. For example, it appears that older adults' slower shift is affected by a retrieval strategy aversion, even for well-learned items, which is related to lower memory ability confidence (Touron \& Hertzog, 2004a, 2004b). Older adults typically rate their current memory abilities lower than do younger adults, and also rate them lower than their own abilities as younger adults (see Hertzog \& Hultsch, 2000). Since strategy choice differences influence age differences in strategy performance, observed age differences in task performance overestimate the degree of an associative learning deficit in older adults (see Rogers et al., 2000). The present examination of age differences in item-level strategy shift patterns facilitates a more comprehensive articulation of how subjective choice influences strategic skill acquisition in older adults, and also clarifies more fundamental properties of strategy shift.

One issue that can be explored through item-level analysis is the quality of strategy shift for each stimulus item - whether the transition occurs abruptly or gradually. Individuals might shift to retrieval only after gathering sufficient knowledge or memory confidence, and never go back to scanning, or they might vacillate between strategies. Age differences in the transition length of strategy shift for each stimulus item might involve the same subjective choice factors that have been implicated previously in NP task performance. For example, older adults' lower memory ability confidence might lead to more reversions to the scanning strategy and to a protracted strategy transition. Strategy reversions might also sporadically occur due to transient memory retrieval failures, although such failures cannot fully account for age differences in strategy shift (see Touron \& Hertzog, 2004a). Although most current quantitative skill acquisition models can account for vacillations in the success of memory retrieval, none consider metacognitive influences on performance.

A second issue that can be explored through item-level analysis is the collective versus variable nature of strategy shift between items. Individuals might shift to retrieval for all items at roughly the same point in training, or might shift to the memory retrieval strategy separately for each item. At least one effort (Haider \& Frensch, 2002) has concluded that strategy shifts can occur simultaneously across items. However, because most skill acquisition models require that the transition occurs separately for each stimulus item (e.g., Logan, 1988; Rickard, 1997), resolution of this issue is significant for theoretical development (see Rickard, 2004). Furthermore, age and individual differences in subjective strategy choice might influence the variability of item-level strategy shifts. For example, older adults might require stronger and more global evidence of their memory ability to confidently choose the retrieval strategy, and therefore shift strategy only after all items are well learned, whereas younger adults shift immediately upon learning each item.

Although much attention has been paid to strategy shifts on the aggregate level, little is known about itemlevel changes in performance. One notable exception, a study by Rickard (2004), demonstrated that item-level RT data from younger adults exhibited step-function decreases that were better predicted by fits to his component power laws (CMPL) model than by the smooth speedup functions that govern parallel execution models (such as the instance model, Logan, 1988, and the exemplar-based random walk model, Palmeri, 1997). Rickard's data indicated separate rather than collective shifts for younger adults - because the point in training at which step-function decreases occurred varied substantially by item-but they were not conclusive regarding the occurrence of strategy shift reversions. The current version of the CMPL model requires that strategy shifts occur in a single step, with no reversions to the algorithm following the transition to retrieval. Although multiple strategy shifts might have compromised Rickard's item-level CMPL fits, no direct test of strategy shift reversions has been conducted to date.

The present study further examines the variability of item strategy shifts, and formally tests the prevalence of strategy shift reversions. Furthermore, we explore the extent to which qualitative age differences exist in itemlevel strategy performance. Older adults' aggregate data indicate greater conservatism in strategy selection, and we anticipate that this conservatism will also lead to more gradual strategy shift transitions for individual items as well as to less between-item variability in strategy shifting (i.e., more collective strategy shifts).

For each item, the transition path from scanning to retrieval was examined using the following measures: (1) initial retrieval strategy use, (2) final scanning strategy use, (3) the total transition space (number of item exposures) between initial retrieval use and final scanning use, (4) the total number of strategy shifts (i.e., from scanning to retrieval or from retrieval to scanning), and (5) the percentage of trials with a single-step transition (i.e., no reversions to scanning following item retrieval). To determine the variability between item shifts, we inspected and compared within-participants standard deviations for the first three measures listed above: initial retrieval, final scanning, and total transition space.

\section{METHOD}

\section{Design}

The primary within-participants independent variable in this study was practice (noun pair repetitions); the primary betweenparticipants independent variable was age (younger or older). Data were also collected for three different between-participants instruction conditions, but the present study was collapsed over condition because no statistically significant condition differences in aggregate or item-level performance indices were obtained. ${ }^{2}$ The dependent variables measured included changes in RT, accuracy, and strategy usage across standard NP task practice, as well as recognition memory RTs, accuracy, and confidence judgments collected follow- 
ing the standard NP task. The present investigation concentrates on trial-level strategy reports.

\section{Participants}

The study included 67 younger adults between the ages of 18 and 24 years and 62 older adults between the ages of 59 and 75 years. The younger adults were undergraduate volunteers participating for extra credit. The older adults were recruited from the community, and received a modest honorarium for their participation. The older adults were sufficiently healthy to visit our on-campus laboratory, and were screened for postsecondary education.

Table 1 reports means and standard errors for participant characteristics. The older adults scored higher on the Shipley Vocabulary Test (Zachary, 1986) than did the younger adults. The younger adults scored higher on the WAIS-R Digit-Symbol subtest (a speeded task of paired-associates matching; Wechsler, 1981) and on a test of digitsymbol memory following the subtest. Obtained age differences were comparable with those typically reported in the literature.

\section{Procedure}

A Visual Basic 6.0 program controlled stimulus presentations and response recordings. Stimuli were presented in 15-point Arial font at a resolution of $1,024 \times 768$ pixels. The participants were seated at a height and distance that optimized their screen viewing and comfort.

Noun pair matching trials began with a centrally presented fixation, followed by a centrally presented target item (e.g., cat-market). These two words were paired horizontally and separated by spaces and a dash. A table of 12 noun pairs was presented at the top of the screen. The pairings did not change, with the location of each pair in the table randomly selected on each trial. Half of the trials presented a target pair matching a pair in the lookup table. The remaining nonmatched trials paired a left-hand word from one pair with a randomly selected right-hand word from a different pair.

After a short set of questionnaires and cognitive ability tests, the participants completed self-paced computer instruction, and then began the noun pair task. Across training, all of the participants received 60 repetitions per item, organized into 30 blocks containing 2 repetitions per item. The participants were instructed to press a key labeled $\mathrm{Y}$ if the target pair had a match in the lookup table, or a key labeled $\mathrm{N}$ if it did not. Strategy reports were obtained following each trial. The participants were instructed to indicate which of the following strategies they used on the previous trial: (1) scan, (2) memory, (3) both, or (4) other, by pressing a key labeled with the first letter of the response strategy. Trial-level error feedback followed each strategy probe. ${ }^{3}$

The participants were offered a rest break following each block, during which they received mean RT and accuracy feedback for the preceding block. The participants were instructed to maintain accuracy near $95 \%$. To prevent fatigue, mandatory 1 -min breaks were enforced every 10 blocks. Following the computer testing, the participants completed a posttest survey, including self-ratings of global memory confidence ("Were you confident to use your memory?" 1 = yes, $5=n o) \cdot{ }^{4}$ As expected, the younger adults were more confident in their memory ability following the noun pair task. $\left(M_{\text {young }}=1.65, S E_{\text {young }}=0.13 ; M_{\text {old }}=3.02, S E_{\text {old }}=0.20\right)$. The younger adults typically required about $2 \mathrm{~h}$ for testing; the older adults typically required about $2.5 \mathrm{~h}$.

\section{RESULTS AND DISCUSSION}

Although the strategy report methodology has been previously supported for aggregate analyses, the significance of report validity for the item-level approach demands corroboration from the present data. RT and error data are presented separately for reported scanning and retrieval trials in Table 2.5 Strategy self-reports appear to be a valid
Table 1 Means (With Standard Errors) of Participant Characteristics

\begin{tabular}{|c|c|c|c|c|}
\hline \multirow[b]{2}{*}{ Measure* } & \multicolumn{2}{|c|}{ Younger } & \multicolumn{2}{|c|}{ Older } \\
\hline & $M$ & $S E$ & $M$ & $S E$ \\
\hline Age (years) & 19.34 & 0.17 & 67.55 & 0.61 \\
\hline Education & 13.46 & 0.16 & 16.24 & 0.36 \\
\hline No. medications & 0.39 & 0.13 & 3.29 & 0.30 \\
\hline Vocabulary & 30.07 & 0.44 & 35.39 & 1.22 \\
\hline Digit-Symbol & 64.81 & 1.24 & 45.57 & 1.52 \\
\hline D-S memory & 7.87 & 0.20 & 4.39 & 0.31 \\
\hline
\end{tabular}

Note-No. medications, self-reported number of daily medications; Vocabulary, number correct out of 40; Digit-Symbol, WAIS-R Digit-Symbol subtest; D-S memory, symbol memory following D-S subtest. "For age comparison, $p<.05$.

index of strategy performance for both younger and older adults. Reported scanning trials were markedly slower $\left[F(1,124)=311.33, M S_{\mathrm{e}}=707,470, p<.01\right]$, varied more in speed $\left[F(1,121)=56.35, M S_{\mathrm{e}}=292,443, p<\right.$ $.01]$, and were more accurate $\left[F(1,127)=100.63, M S_{\mathrm{e}}=\right.$ $8.02, p<.01]$ than retrieval responses. As expected, age differences were noted for the RT measures but not for accuracy (per instructions).

As is typically found, the younger adults relied on the retrieval strategy more often during training $[F(1,118)=$ $\left.46.64, M S_{\mathrm{e}}=19,353, p<.01\right]$, an age difference that persisted through the final block of training $\left[M_{\text {young }}=97.34\right.$, $S E_{\text {young }}=3.40 ; M_{\text {old }}=65.99, S E_{\text {old }}=3.51 ; F(1,126)=$ $\left.41.11, M S_{\mathrm{e}}=764, p<.01\right]$. For each age group, the course of strategy shift across items is shown in Figure 1.

\section{Timing of Strategy Shift}

To assess age differences in item-level strategy shift patterns, the commencement of retrieval and the conclusion of scanning were compared across age groups (see Table 3). Age differences in overall strategy use were not determined by the timing of initial retrieval attempts, because both younger and older adults began retrieving after about seven item exposures $\left[F(1,126)=0.20, M S_{\mathrm{e}}=52\right.$, $p=.66]$. Instead, age differences in strategy use seemed to be determined by reluctance to abandon use of the scanning strategy. Although younger adults' final scanning generally occurred midway through training (out of 60 total exposures), older adults persisted in scanning much longer $\left[F(1,126)=64.00, M S_{\mathrm{e}}=206, p<.01\right]$.

Given the outcomes above, it directly follows that the transition space between initial retrieval and final scanning was shorter for the younger adults than for the older adults

Table 2

Performance Means (With Standard Errors) by Strategy and Age

\begin{tabular}{lcrrrcc}
\hline & $\mathrm{RT}$ & $S E^{\mathrm{A}, \mathrm{B}}$ & $S D$ & $S E^{\mathrm{A}, \mathrm{B}}$ & $\%$ Accuracy & $S E^{\mathrm{B}, \mathrm{C}}$ \\
\hline SCAN $_{\text {young }}$ & 2,903 & 49 & 1,033 & 56 & 99.6 & 0.08 \\
SCAN $_{\text {old }}$ & 4,027 & 42 & 1,246 & 96 & 98.5 & 0.34 \\
$\mathrm{RET}_{\text {young }}$ & 1,012 & 10 & 418 & 23 & 94.4 & 0.51 \\
$\mathrm{RET}_{\text {old }}$ & 2,246 & 27 & 856 & 99 & 96.6 & 0.62 \\
\hline
\end{tabular}

AFor age comparison, $p<.05$. BFor strategy comparison, $p<$ .05 . CFor age $\times$ strategy interaction, $p<.05$. 


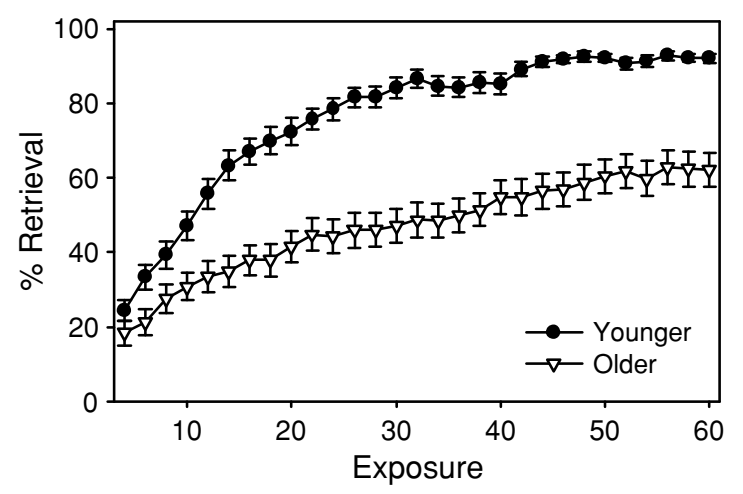

Figure 1. Mean percentage retrieval strategy use by age and number of stimulus exposures.

$\left[F(1,126)=59.43, M S_{\mathrm{e}}=209, p<.01\right]$. For both younger adults and older adults, nevertheless, the average strategy transition periods were quite prolonged, countering theoretical assumptions that item-level strategy shifts should occur abruptly. Indeed, a single-step strategy transition (measured as percentage of items), as is typically assumed, was not the dominant pattern for the younger adults or the older adults, although the younger adults shifted more quickly than the older adults $\left[F(1,126)=7.15, M S_{\mathrm{e}}=314, p<\right.$ $.01]$. When single-step strategy transitions did not occur, both the younger and older adults made several strategy reversions. As hypothesized, the younger adults made fewer strategy shift reversions than the older adults $[F(1,126)=$ $\left.21.17, M S_{\mathrm{e}}=46, p<.01\right]$. Distributions of strategy reversions are depicted in Figure 2 for each age group.

Associations of item-level strategy shift patterns with final retrieval reliance and memory ability confidence were examined via correlations for the combined sample as well as separately for younger and older adults (see Table 4). The outcomes support the conclusion that the age discrepancy in strategy reliance is determined by reluctance to abandon scanning rather than by reluctance to attempt retrieval. Both overall retrieval use and memory ability confidence at the end of training were not significantly related to the timing of retrieval commencement, although both were strongly related to the cessation of scanning and to the total transition space. When the cor-

Table 3

Means (With Standard Errors) of Item-Level Measures by Age Group

\begin{tabular}{lccccc}
\hline \multirow{2}{*}{\multicolumn{1}{c}{ Measure }} & \multicolumn{2}{c}{ Younger } & & \multicolumn{2}{c}{ Older } \\
\cline { 2 - 3 } \cline { 5 - 6 } & $M$ & $S E$ & & $M$ & $S E$ \\
\hline First retrieval & 7.18 & 0.89 & & 7.75 & 0.92 \\
Last scanning* & 25.94 & 1.77 & & 46.24 & 1.82 \\
Transition space* & 18.75 & 1.78 & & 38.49 & 1.84 \\
Number of shifts* & 7.37 & 0.84 & & 12.9 & 0.86 \\
One-step transitions* & 11.87 & 2.18 & & 3.49 & 2.25 \\
First retrieval $S D^{*}$ & 2.63 & 0.41 & & 3.49 & 2.25 \\
Last scanning $S D^{*}$ & 10.4 & 0.65 & & 6.0 & 0.67 \\
Transition space $S D^{*}$ & 10.95 & 0.61 & & 8.37 & 0.63 \\
\hline "For age comparison, $p<.05$. & & & &
\end{tabular}

relations were examined by age group, the obtained relationship between confidence and the timing of scanning cessation for the combined sample appeared to be driven by age differences and a strong relationship within the older adult sample. It is not surprising that the participants who failed to abandon the scanning strategy and therefore transitioned to retrieval slowly were less confident and retrieved less at the end of training. It is important to recognize, however, that no continuing advantage or disincentive was apparent for the participants who attempted retrieval early in training. A higher frequency of singlestep strategy transitions correlated with greater reliance on retrieval at the end of training, as well as with higher memory ability confidence, but these relationships appeared to be driven primarily by age differences in performance. When multiple strategy shifts did occur, more frequent shifting was associated with lower memory confidence for the younger adults, but did not translate into a disadvantage in retrieval reliance at the end of training.

To ensure that the relationships between item-level performance and memory confidence were not confounded with general cognitive ability, strategy shift indices were also correlated with vocabulary and digit-symbol scores. Generally, cognitive abilities could not account for itemlevel strategy shift patterns $(p s>.05)$. Slower processing speed was associated with more frequent shifts by the younger adults $(r=.27, p<.05)$; high vocabulary was associated with earlier initial retrieval attempts by the older adults $(r=.27, p<.05)$.

\section{Strategy Shift Variability}

To assess the extent to which item-level strategy shifts occurred individually as opposed to collectively, we examined within-participants standard deviations of itemlevel strategy shift indices (see Table 3). Where standard deviations were statistically indistinguishable from zero, this was taken as support for a collective strategy transition across items.

Commencement of retrieval strategy use varied between items for both younger and older adults. The younger adults' commencement of retrieval was less variable than that of older adults $\left[F(1,126)=6.82, M S_{\mathrm{e}}=11, p=.01\right]$, counter to our expectations. Variability across items was also significant for abandonment of scanning, although in this case, the younger adults displayed more variability across items than the older adults $\left[F(1,126)=22.42, M S_{\mathrm{e}}=46\right.$, $p<.01]$. This comparison seems more critical, because we earlier demonstrated that age differences in overall strategy shift were more strongly determined by scanning cessation than by the timing of initial retrieval attempts. In terms of the total transition space, variability was still significant, and was again more pronounced for younger adults than for older adults $\left[F(1,126)=8.78, M S_{\mathrm{e}}=24, p<.01\right]$. Although older adults' strategy shifts do not seem fully collective, they generally exhibit less variability between items than do strategy shifts made by younger adults.

The relationship between final retrieval reliance and the variability of item-level strategy shifts was also examined via correlations for the combined sample and 


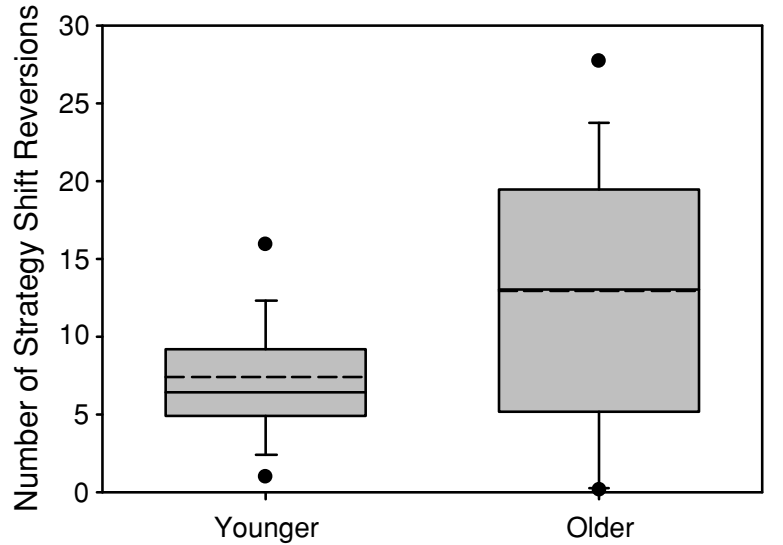

Figure 2. Boxplots showing the number of strategy shift reversions for younger and older adults. Mean levels are indicated by dashed lines, and circles indicate the 5th and 95th percentiles.

each age group (see Table 4). Variability between items in the timing of initial retrieval attempts was associated with low retrieval use and low memory ability confidence, but these relationships appeared to be driven primarily by age differences in performance. Variability in final scanning was associated with high retrieval use and high memory ability confidence, particularly for older adults. Older adult participants whose item-level data varied in the total transition space relied more on retrieval at the end of training. It appears that metacognitive underconfidence and conservatism might lead to more collective strategy shift performance, particularly in the final shift decision. Correlations of strategy shift indices with vocabulary and digit-symbol scores were again computed in order to assess the influence of general cognitive ability; none of these relationships were significant ( $p \mathrm{~s}>.05)$.

\section{CONCLUSIONS}

The item-level approach to strategy shift analysis provides a more comprehensive account of the skill acquisition process. Item-level strategy shifts in the noun pair task are neither abrupt nor collective. Instead, it appears that participants generally shift to retrieval on a discriminate item-by-item and trial-by-trial basis as a consequence of their current levels of knowledge and memory confidence. Item-level strategy shifts do not appear to occur in a single step (as assumed by the CMPL model; Rickard, 2004), with shift reversions as the exception rather than the rule. Earlier and shorter strategy transitions were found, however, to be associated with higher memory confidence and with superior performance, indicating that earlier stepwise shift assumptions might characterize ideal rather than authentic behavior. Task characteristics might also influence the abruptness of item-level strategy shift (see Touron \& Hertzog, 2004b, for a demonstration of task effects using aggregate data), and account in part for diverging outcomes.

For all shift indices, item-level variability was pronounced, arguing against a collective account of strategy shift. Perhaps it is the case that the quality of the strategy shift is instrumental to the variability of item strategy shifts. Haider and Frensch (2002) found evidence for collective strategy shifts in their alphabet verification task, in which transitions are characterized by the recognition and implementation of a selective attention strategy. After the attention strategy has been learned, it can be applied to any stimuli, repeated or novel, indiscriminately. In tasks such as the NP task, in which strategy transitions require the learning of individual stimuli, simple comprehension of the strategy cannot lead to successful strategy implementation on a global level until all items have been learned to a reasonable criterion. Such task-based explanations are potentially important but do not provide a comprehensive understanding of the issue, given the present study's exposure of age and individual differences in item-level shift variability within a single task. That these differences are related to metacognitive variables rather than cognitive ability suggests that the extent to which participants shift collectively is under strategic control.

Age and individual differences in item-level strategy shift patterns are pronounced, and suggest that performance is affected by subjective metacognitive factors above cognitive influences such as associative learning deficits and general slowing. Older adults are slower to transition to memory retrieval in the NP tasks due to a reluctance to abandon the scanning strategy, a tendency to switch frequently between strategies for a given item, and a greater inclination toward collective shifting - patterns associated with low memory confidence.

Table 4

Correlations of Item-Level Measures With Retrieval Use and Memory Ability Confidence at the End of Training for the Combined Sample and Separately for Younger and Older Adults

\begin{tabular}{|c|c|c|c|c|c|c|}
\hline Measure & Retrieval $_{\text {all }}$ & Confidence $_{\text {all }}$ & Retrieval $_{\text {young }}$ & Confidence $_{\text {young }}$ & Retrieval $_{\text {old }}$ & Confidence $_{\text {old }}$ \\
\hline First retrieval & -.02 & -.03 & -.06 & -.08 & .02 & -.06 \\
\hline Last scanning & -.72 & .47 & -.50 & .19 & -.71 & .32 \\
\hline Transition space & -.72 & .49 & -.50 & .25 & -.69 & .33 \\
\hline Number of shifts & -.15 & .23 & -.07 & .32 & -.07 & -.09 \\
\hline One-step transitions & .24 & -.18 & .12 & -.14 & .22 & .15 \\
\hline First retrieval $S D$ & -.27 & .21 & -.01 & .15 & -.22 & .10 \\
\hline Last scanning $S D$ & .60 & -.26 & .21 & .16 & .67 & -.33 \\
\hline Transition space $S D$ & .38 & -.12 & .23 & .19 & .37 & -.19 \\
\hline
\end{tabular}

Note-Boldface type, $p<.05$. 


\section{REFERENCES}

Ackerman, P. L., \& Woltz, D. J. (1994). Determinants of learning and performance in an associative memory/substitution task: Task constraints, individual differences, volition, and motivation. Journal of Educational Psychology, 86, 487-515.

Bosman, E. A., \& Charness, N. (1996). Age-related differences in skilled performance and skill acquisition. In F. Blanchard-Fields \& T. M. Hess (Eds.), Perspectives on cognitive change in adulthood and aging (pp. 428-453). New York: McGraw-Hill.

Charness, N. (1981). Aging and skilled problem solving. Journal of Experimental Psychology: General, 110, 21-38.

Dunlosky, J., \& Hertzog, C. (2001). Measuring strategy production during associative learning: The relative utility of concurrent versus retrospective reports. Memory \& Cognition, 29, 247-253.

Estes, W. K., \& MadDox, W. T. (2005). Risks of drawing inferences about cognitive processes from model fits to individual versus average performance. Psychonomic Bulletin \& Review, 12, 403-408.

FISK, A. D., \& Rogers, W. A. (1991). Toward an understanding of agerelated memory and visual search effects. Journal of Experimental Psychology: General, 120, 131-149.

HAider, H., \& Frensch, P. A. (2002). Why aggregated learning follows the power law of practice when individual learning does not: Comments on Rickard (1997, 1999), Delaney et al. (1998), and Palmeri (1999). Journal of Experimental Psychology: Learning, Memory, \& Cognition, 28, 392-406.

Hertzog, C., \& Hultsch, D. F. (2000). Metacognition in adulthood and old age. In F. I. M. Craik \& T. A. Salthouse (Eds.), The handbook of aging and cognition (2nd ed., pp. 417-466). Mahwah, NJ: Erlbaum.

Hoyer, W. J., Cerella, J., \& ONyper, S. V. (2003). Item learning in cognitive skill training: Effects of item difficulty. Memory \& Cognition, 31, 1260-1270.

Lemaire, P., \& Arnaud, L. (2002, April). Age-related changes in strategy adaptivity: Insights from computational estimation. Poster presented at the Cognitive Aging Conference, Atlanta, GA.

Light, L. L. (1996). Memory and aging. In E. L. Bjork \& R. A. Bjork (Eds.), Memory: Handbook of perception and cognition (2nd ed., pp. 443-490). San Diego: Academic Press.

LogAn, G. D. (1988). Toward an instance theory of automatization. Psychological Review, 95, 492-527.

Myung, I. J., Kim, C., \& PitT, M. A. (2000). Toward an explanation of the power law artifact: Insights from response surface analysis. Memory \& Cognition, 28, 832-840.

PAlmeri, T. J. (1997). Exemplar similarity and the development of automaticity. Journal of Experimental Psychology: Learning, Memory, \& Cognition, 23, 324-354.

RICKARD, T. C. (1997). Bending the power law: A CMPL theory of strategy shifts and the automatization of cognitive skills. Journal of Experimental Psychology: General, 126, 288-311.

RICKARD, T. C. (2004). Strategy execution in cognitive skill learning: An item-level test of candidate models. Journal of Experimental Psychology: Learning, Memory, \& Cognition, 30, 65-82.

Rogers, W. A., \& Gilbert, D. K. (1997). Do performance strategies mediate age-related differences in associative learning? Psychology \& Aging, 12, 620-633.

Rogers, W. A., Hertzog, C., \& Fisk, A. D. (2000). An individual differences analysis of ability and strategy influences: Age-related differences in associative learning. Journal of Experimental Psychology: Learning, Memory, \& Cognition, 26, 359-394.

Salthouse, T. A. (1991). Theoretical perspectives on cognitive aging. Hillsdale, NJ: Erlbaum.
Salthouse, T. A. (1994). The nature of the influence of speed on adult age differences in cognition. Developmental Psychology, 30, 240259.

Salthouse, T. A., \& Dunlosky, J. (1995). Analyses of adult age differences in associative learning. Zeitschrift für Psychologie, 203, 351360 .

Shiffrin, R. M., \& Schneider, W. (1977). Controlled and automatic human information processing: II. Perceptual learning, automatic attending, and a general theory. Psychological Review, 84, 127-190.

Siegler, R. S., \& Lemaire, P. (1997). Older and younger adults' strategy choices in multiplication: Testing predictions of ASCM using the choice/no-choice method. Journal of Experimental Psychology: General, 126, 71-92.

Strayer, D. L., \& Kramer, A. F. (1994). Aging and skill acquisition: Learning-performance distinctions. Psychology \& Aging, 9, 589605.

Touron, D. R., \& Hertzog, C. (2004a). Distinguishing age differences in knowledge, strategy use, and confidence during strategic skill acquisition. Psychology \& Aging, 19, 452-466.

Touron, D. R., \& Hertzog, C. (2004b). Strategy shift affordance and strategy choice in young and older adults. Memory \& Cognition, 32, 298-310.

Touron, D. R., Hoyer, W. J., \& Cerella, J. (2001). Cognitive skill acquisition and transfer in younger and older adults. Psychology \& Aging, 16, 555-563.

Touron, D. R., Hoyer, W. J., \& Cerella, J. (2004). Cognitive skill learning: Age-related differences in strategy shifts and speed of component operations. Psychology \& Aging, 19, 565-580.

WeCHSLER, D. (1981). Wechsler Adult Intelligence Scale-Revised. New York: Psychological Corporation.

ZACHARY, R. A. (1986). Shipley Institute of Living Scale: Revised manual. Los Angeles: Western Psychological Services.

\section{NOTES}

1. The extent to which task performance is affected by strategy reporting is less clear. Although Rickard (2004) demonstrated a younger adult bias toward retrieval when strategy reports were collected, Touron et al. (2004) found that strategy reporting did not influence task performance for younger or older adults.

2 . Equal numbers of younger and older adults were randomly assigned to a standard instruction condition, a condition that instructed participants to value speed over accuracy, or a condition that explicitly instructed participants to use the memory retrieval strategy as much as possible.

3. Following completion of the standard NP task, participants completed four blocks of recognition memory probes. Following each memory probe, confidence judgments were collected. These memory probe and trial-level confidence data are not the focus of the present investigation.

4. Participants provided judgments of learning globally and for each noun pair item, scaled in decades, as well as for a cued-recall task. They also provided verbal descriptions of their strategy use, and completed self-ratings of global memory confidence, expected memorization, effort, and perceived improvement.

5. Because the "both" and "other" strategy categories were reported infrequently (on less than $5 \%$ of trials, which is typical for the NP task; Touron \& Hertzog, 2004b), these categories were not examined further.

(Manuscript received October 24, 2005; revision accepted for publication March 16, 2006.) 